\title{
Imaging in Diffuse Media using Pulsed-Ultrasound-Modulated Light and the Photorefractive Effect
}

\author{
Lei Sui ${ }^{1}$, Ronald A. Roy ${ }^{1}$, Charles A. DiMarzio ${ }^{2}$, and Todd W. Murray ${ }^{1}$ \\ ${ }^{1}$ Department of Aerospace and Mechanical Engineering, Boston University \\ 110 Cummington Street, Boston, MA 02215 \\ ${ }^{2}$ Department of Electrical and Computer Engineering, Northeastern University \\ 360 Huntington Avenue, Boston, MA 02115
}

Acousto-optical imaging in diffuse media is a dual wave sensing technique in which an acoustic field interacts with multiply scattered laser light. The acoustic field causes a phase modulation in the optical field emanating from the interaction region, and this phase modulated optical field carries with it information about the local opto-mechanical properties of the media. In this work, a pulsed ultrasound transducer is used to modulate the optical field and a photorefractive-crystal based interferometry system is used for the detection of ultrasound-modulated light. The use of short pulses of focused ultrasound allows for one-dimensional AO image to be obtained along the transducer axis from a single, time averaged acousto-optical signal. The axial and lateral resolutions of the system are controlled by the spatial pulse length and width of the ultrasound beam, respectively. In addition, twodimensional images of optical inhomogeneities buried in turbid media are obtained through scanning of the ultrasound transducer in one dimension.

OCIS codes: $170.3880,110.7050,120.3180,110.7170$. 


\section{INTRODUCTION}

Optical techniques for imaging in turbid media, such as biological tissues, have received considerable attention in recent years. ${ }^{1}$ This is due to both the non-invasive and non-ionizing properties of these methods, as well as considerable optical contrast observed, for instance, between normal and cancerous tissues. One of the major difficulties in purely optical imaging is that light is highly scattered in biological tissues, which typically results in a trade-off between the imaging resolution and the imaging depth. A technique that holds promise in improving the resolution at greater depth makes use of a combination of diffuse laser light and focused ultrasound and takes advantage of the fact that ultrasound is much less readily scattered in biological tissues. In this technique, a focused ultrasound beam is used to modulate or "tag" diffuse light through the displacement of optical scatterers and ultrasound-induced changes in refractive index. $^{2}$ The detection of this tagged light yields spatially resolved opto-mechanical information. The advantage of this hybrid technique is that it can reveal the optically relevant physiological information while maintaining ultrasonic spatial resolution.

Marks et $a l^{3}$ first reported the modulation of diffuse laser light with pulsed focused ultrasound in a homogeneous scattering media in 1993. This was closely followed by the work of Wang et al. ${ }^{4}$ and Kempe et al. ${ }^{5}$ who demonstrated the utility of using the tagging of diffuse photons for imaging purposes. In order to enhance the signalto-noise ratio (SNR), the former group employed a continuous-wave $(\mathrm{CW})$ ultrasound source, and the modulated signals were detected using a photomultiplier tube (PMT). Conventional single detector techniques result in extremely low light levels when the detection aperture is limited to single speckle detection, and reduced modulation depth 
when the detection aperture is increased to receive multiple speckles. To overcome the limitations of single detector techniques, Leveque et al. ${ }^{6}$ employed a multiple detector system based on a CCD array. By adding up the individual modulation amplitudes from all of the pixels, they improved the SNR by a factor of $\sim \mathrm{N}^{1 / 2}$, where $\mathrm{N}$ is the number of coherence areas detected, corresponding to the number of pixels on the CCD. The CCD approach that has been employed is not suitable for pulsed ultrasound measurements due to the relatively long time required for image acquisition. In addition, the system is sensitive to speckle decorrelation during the measurement time. More recently, Gross et $a l^{7}$ improved the sensitivity of this parallel detection scheme by using a heterodyne technique, and proposed to filter out the speckle decorrelation noise through a spatial filter system.

In the majority of acousto-optical imaging systems, $\mathrm{CW}$ ultrasound sources are employed, which allow for greatly enhanced sensitivity and noise immunity through a reduction in detection bandwidth. The use of broad-band pulsed ultrasound, however, provides two important advantages over $\mathrm{CW}$ ultrasound: enhanced spatial resolution is easily achieved along the ultrasonic axis, and the deleterious bio-effects that can result from the high-intensity ultrasound exposure can be minimized. To achieve axial resolution for $\mathrm{CW}$ exposures, Wang and $\mathrm{Ku}^{8}$ introduced a technique in which a single optical detector is used and the CW ultrasound source is chirped, thereby assigning a particular frequency to each location along the ultrasonic axis. A 1-D axial scan could then be produced from the time-dependent frequency-domain information of the ultrasound-modulated signals. Yao et al. ${ }^{9}$ and Forget et al. ${ }^{10}$ also combined this technique with the parallel detection system to achieve the axial resolution with enhanced 
sensitivity. Alternatively, in time domain, Lev and $\mathrm{Sfez}^{11}$ used ultrasonic pulses to construct $\mathrm{CW}$ signals by devising a reshaping algorithm to synchronize the ultrasonic pulses. Recently, we have designed a photorefractive-crystal (PRC) based interferometry system for the detection of ultrasound-modulated light in diffuse media, which was shown to have sufficient sensitivity to detect the transiently modulated optical signals generated using a pulsed ultrasound source operating at biomedically relevant output levels. ${ }^{12-13}$

In this paper, the imaging capability of our PRC based detection system is demonstrated in optically diffuse tissue phantoms with imbedded targets. By using short pulses of focused ultrasound, a 1-D image of the target along the ultrasonic axis may be obtained through a single (averaged) time domain waveform. The transverse resolution of the measurement is determined by the width of the ultrasonic beam, while the axial resolution is controlled by the spatial length of the ultrasound pulse. In addition, twodimensional imaging of optical inhomogeneities buried in turbid media is demonstrated through scanning of the ultrasound transducer in one dimension.

\section{INTERFEROMETRIC DETECTION SYSTEM}

\section{A. Background and Theoretical Considerations}

PRC based interferometers have seen widespread use in the optical detection of ultrasound in nondestructive evaluation and materials characterization applications. ${ }^{14-16}$ These systems are ideally suited for detecting high frequency vibrations from optically rough surfaces. In such a system, the signal beam is reflected off of the target material acquiring a phase modulation in the presence of motion. The scattered signal beam is then sent to the PRC where it is mixed with a reference beam (or pump beam). An 
interference pattern is formed in the crystal exciting free carriers in the bright regions, which drift or diffuse to the dark regions leading to a space charge field formation. The index of refraction is modulated through the electro-optic effect, and the reference beam is diffracted off of this grating into the signal beam direction in the two-wave mixing (TWM) process. An external electric field can be applied to the crystal, which serves to enhance the TWM gain and hence the detection sensitivity. ${ }^{17}$ The diffracted reference beam's phase front replicates that of the signal beam, providing a local oscillator (LO). The diffracted reference beam and transmitted signal beam interfere at the photodetector where any phase modulation encoded on the signal beam is converted to an intensity modulation. It should be mentioned that the PRC is adaptive in that the index grating is continually "rewritten" on the time scale of the PRC response time, while high frequency modulation produced by the ultrasonic source is not compensated for, producing a relative phase shift between the signal and reference beams and an intensity change at the detector. The PRC response time represents the time required for space charge field formation, and it is controlled by both the material parameters of the PRC and the intensity of the incident beams. For a given crystal, the response time typically varies inversely with the power density incident on the crystal. ${ }^{18}$ The adaptive property of the PRC allows the detection systems to partly compensate for low frequency shifts in the speckle pattern due to environmental vibration and physiological motion.

The operating principles of the PRC based interferometers have been described in the literature. ${ }^{17-20}$ Here we consider the case of two plane waves interfering within a photorefractive crystal as illustrated in Fig. 1. The signal beam intensity is represented by $\mathrm{I}_{\mathrm{SO}}$ at the entrance to the PRC and $\mathrm{I}_{\mathrm{SE}}$ at the exit of the PRC that has a length, $\mathrm{L}$. The 
signal beam is phase modulated through interaction with the ultrasound and the phase modulation has the form:

$$
\phi=\phi_{a} f(t) \sin \left(\omega_{a} t+\chi_{r}\right),
$$

where $\phi_{a}$ and $\omega_{a}$ are the amplitude and the angular frequency of the phase modulation, respectively, $f(t)$ is the normalized envelope of the phase modulation (corresponding to the envelope of the ultrasonic tone burst in this plane-wave case), and $\chi_{r}$ is a constant that depends on the optical path. It is assumed that the signal beam is phase modulated at a frequency sufficiently high so that the PRC response time is long with respect to the oscillation period. Furthermore, it is assumed that the length of the ultrasonic tone burst, as defined by the envelope function $f(t)$, is also short with respect to the crystal response time such that the index grating remains static over the measurement period. Under our experimental condition, the response time of the PRC is approximately $150 \mathrm{~ms}$, the acoustic period is $1 \mu \mathrm{s}$, and the duration of the acoustic pulse is typically less than $10 \mu \mathrm{s}$; these conditions support the assumptions listed above.

The signal beam is amplified as it propagates through the crystal with a two wave mixing gain of $\gamma$ as the reference beam is diffracted into the signal beam direction. The diffracted reference beam has the same phase front as the transmitted signal beam, but does not acquire the high frequency phase modulation. Note that the gain coefficient is complex and the diffracted reference beam may be uniformly shifted in phase with respect to the signal beam. The gain coefficient is given by $\gamma=\gamma^{\prime}+i \gamma^{\prime \prime}$, where $\gamma^{\prime}$ is the real part of the gain and $\gamma^{\prime \prime}$ is the imaginary part. The optical absorption coefficient in the crystal is given by $\alpha$. In the undepleted pump approximation where the intensity of the 
reference beam, denoted by $I_{R}$ in Fig. 1, is large compared to that of the signal beam, the intensity of the transmitted signal beam at the exit of the crystal, $\mathrm{I}_{\mathrm{SE}}$, is given by ${ }^{19}$

$$
I_{S E}=\exp (-\alpha L) I_{S O}\left\{\left|e^{\gamma L}-1\right|^{2}+1+2 \operatorname{Re}\left[\left(e^{\gamma L}-1\right) * \exp \left(i \phi_{a} f(t) \sin \left(\omega_{a} t+\chi_{r}\right)\right)\right]\right\}
$$

where "*" denotes the complex conjugate.

Expanding Eq. (2) using a Bessel series expansion and retaining only the lowest order terms we find:

$$
I_{S E}^{A C}=4 \exp (-\alpha L) I_{S O} e^{\gamma^{\prime} L} \sin \left(\gamma^{\prime \prime} L\right) J_{1}\left(\phi_{a} f(t)\right) \sin \left(\omega_{a} t+\chi_{r}\right)
$$

and

$$
I_{S E}^{D C}=\exp (-\alpha L) I_{S O}\left\{\left|e^{\gamma L}-1\right|^{2}+1+2\left[e^{\gamma^{\prime} L} \cos \left(\gamma^{\prime \prime} L\right)-1\right] J_{0}\left(\phi_{a} f(t)\right)\right\}
$$

Equation 3 gives the intensity of the signal beam modulation at the ultrasound frequency, $\omega_{\mathrm{a}}$. Equation 4 shows that, in addition to the modulation at the ultrasound frequency, a "DC-shifted" signal is expected which depends only on the amplitude of the phase modulation and the envelope of the ultrasound pulse train. The resulting signal at the detector, found by summing Eq. 3 and Eq. 4, is a sinusoidal pulse train of duration $f(t)$ riding on top of a DC offset.

Now consider the case of an ultrasonic pulse propagating in an optically diffuse media. Photons travel over multiple paths from the optical source to the detection system, and the phase modulation induced by the ultrasound depends on the spatial position that the acousto-optical interaction takes place. This is taken into account in Eq. 1 through the variable $\chi_{r}$, which is path dependent. Viable photon paths encompass a region with characteristic dimensions larger than an acoustic wavelength. Thus, when collecting light from multiple optical paths in highly diffuse media, it is expected that $\chi_{r}$ will be 
distributed randomly between 0 and $2 \pi$ and the signals observed at the ultrasonic frequency (given by Eq. 3) from light traveling over different paths are not expected to add coherently at the detector. The signals given by Eq. 4, however, are independent of optical path, and depend only on the amplitude of the phase modulation and the envelope function. This allows for coherent summation of individual photon contributions at the detector. The signals given by Eqs. 3 and 4 are strongly dependent on the photorefractive gain. The modulus of $\gamma$ represents the strength of the diffraction grating inside the crystal and the phase of $\gamma$ is associated with the spatial phase shift between the illumination and index of refraction gratings. In the linear detection of small amplitude signals, photorefractive interferometers are typically designed such that the diffracted reference beam and transmitted signal beam are placed in quadrature. However, in our experiment, it is found that the component of the signal at the ultrasound frequency (Eq. 3) vanishes very quickly with increasing diffusivity compared to the DC offset signal (Eq. 4), and it is the latter component that we propose to use for sensing and imaging applications. In order to maximize this signal, the PRC based interferometer configuration shown in Fig. 2 is chosen, where the diffracted reference beam and transmitted signal beam are in phase giving pure (real) photorefractive gain. In this case, the intensity of the signal at the ultrasound frequency goes to zero. While this configuration is relatively insensitive to small phase modulations, the phase modulation induced by the ultrasound in our experiments is found to be large enough to contribute to a DC offset signal with an adequate sensitivity for imaging.

\section{B. Experimental Setup}


The experimental setup is shown in Fig. 2. A reference coordinate system is given, with the $\mathrm{Z}$ axis corresponding to the ultrasonic axis, the $\mathrm{Y}$ axis indicating the optical axis, and the $\mathrm{X}$ axis being perpendicular to both the acoustic and optical axes. The output of a frequency doubled Nd:YAG laser source, with $80 \mathrm{~mW}$ power and $532 \mathrm{~nm}$ wavelength, is sent to a variable beam splitter where it is split into signal beam and reference beams with a power ratio of approximately $25: 1$. The reference beam is directed around the test tank and sent directly to the PRC. The signal beam is then sent through a $10 \mathrm{x}$ beam expander to the submerged tissue-mimicking phantom via the flat glass wall of the tank. After the tank, the scattered and ultrasonically modulated light is collected by a lens with an aperture of $5 \mathrm{~cm}$, and a focal length of $10 \mathrm{~cm}$. This collected light is directed into the PRC where it interferes with the reference beam at an angle of $20^{\circ}$. The light incident on the PRC is s-polarized as shown in Figure 1. Our PRC detector employs a BSO crystal with dimensions of $5 \mathrm{~mm} \times 5 \mathrm{~mm} \times 7 \mathrm{~mm}$ along the $\mathrm{X}, \mathrm{Z}$ and $\mathrm{Y}$ axes, respectively, and a holographic cut along the [001], [110] and [1 10$]$ directions. A 4-kHz, $10-\mathrm{kV} / \mathrm{cm}$ peak-to-peak AC field is applied to the crystal to enhance the grating strength and improve the two-wave mixing gain. Under our experimental condition, the photorefractive intensity gain $\Gamma\left(\Gamma=2 \gamma^{\prime}\right)$ is measured to be about $0.25 \mathrm{~cm}^{-1}$. After the PRC, the signal beam and diffracted reference beam (LO that is wave-front-matched to the signal beam) are sent through a lens (aperture $=5 \mathrm{~cm}$, focal length $=10 \mathrm{~cm}$ ) and optical band-pass filter to an avalanche photodiode (APD) with a 10-mm diameter active aperture. The signal from the APD is amplified, low-pass filtered at $500 \mathrm{kHz}$, and sent to a digital storage oscilloscope where the signal is coherently averaged 5000 times. 
The sound source used to modulate the diffuse light is an unbacked, singleelement, spherically focused, piezoelectric transducer (Sonic Concepts). It has a $6.3-\mathrm{cm}$ focal distance and a 7.0-cm aperture. The central frequency of the transducer is $1.1 \mathrm{MHz}$ and the bandwidth is $0.85-1.35 \mathrm{MHz}$. The focal region of the transducer, defined by the full width of half maximum intensity (FWHM), is a cigar-shaped ellipsoid with a long axis of about $9 \mathrm{~mm}$ and a short axis of about $1.5 \mathrm{~mm}$. The peak focal pressure used in our following experiments is approximately $1 \mathrm{MPa}$, measured with a needle hydrophone. The ultrasonic axis is set perpendicular to the laser illumination direction. The transducer is mounted on a 3-D automated translation stage (Velmex), controlled by the computer via a RS-232 port. The transducer is driven using a $1 \mathrm{MHz}$ pulse train. The pulse is produced using a standard function generator, amplified by a fixed-gain power amplifier, and sent to an impedance matching box before the transducer. The pulse repetition frequency used is typically $100 \mathrm{~Hz}$.

A tissue-mimicking gel phantom is submerged in a small glass tank filled with degassed and filtered water. The tank dimensions are $30 \mathrm{~cm} \times 30 \mathrm{~cm} \times 20 \mathrm{~cm}$ along the $\mathrm{X}$, $\mathrm{Y}$ and $\mathrm{Z}$ axes, respectively. The phantom is an acrylamide gel fabricated using the recipe given in Ref. 12. Four hundred nanometer-diameter polystyrene microspheres are added to the gel during fabrication in order to modify the optical scattering coefficient; the particle-free gel is essentially transparent. The sound speed and the density of the phantom are measured to be about $1500 \mathrm{~m} / \mathrm{s}$ and $1050 \mathrm{Kg} / \mathrm{m}^{3}$, matching those found in human breast tissue. ${ }^{21}$ The dimensions of the phantom are about $4 \mathrm{~cm} \times 2.7 \mathrm{~cm} \times 4 \mathrm{~cm}(X$, $\mathrm{Y}, \mathrm{Z})$. A $5 \mathrm{~mm} \times 8 \mathrm{~mm} \times 5 \mathrm{~mm}(\mathrm{X}, \mathrm{Y}, \mathrm{Z})$ optical absorber is imbedded at the center of the phantom. The optical absorber is made using the same recipe as its surrounding phantom 
except that India ink is added to enhance the optical absorption coefficient; the ink has little effect on the acoustic properties of the material. Thus, we obtain a target imbedded in the gel possessing high optical absorption contrast and negligible acoustic contrast. A cut-away view of the phantom used in our experiment is shown in Fig. 3, along with our reference coordinate. The reduced scattering coefficient of the phantom is about $2 \mathrm{~cm}^{-1}$ and the absorption coefficient of the optical absorber is about $3 \mathrm{~cm}^{-1}$.

\section{RESULTS AND DISCUSSION}

Figure 4 shows the normalized pressure waveforms generated by the sound source when driven by different duration electronic pulses (tone bursts) with $1 \mathrm{MHz}$ center frequency, where (a) (b) and (c) correspond to 1-cycle, 2-cycle and 4-cycle pulses, respectively. From Fig. 4, one can clearly see the transducer ring-down effect due to the finite bandwidth of the transducer. Unless otherwise noted, a 2-cycle pulse was employed in the experiments presented here.

In our experiment, the transducer is scanned along $\mathrm{X}$ axis perpendicular to the optical source. Before scanning, the system is aligned to ensure that the center of the phantom roughly coincides with the center of the focal region of the transducer. The expanded signal beam is then directed at the middle of the front surface of the phantom. When the acoustic focal zone is displaced from the optical absorber, the typical ultrasound-modulated signals detected are shown in Fig. 5. The top trace (which has been offset for display purposes) shows the response of the system with the reference beam blocked. The signal beam passes directly through the PRC to the APD, and the

presence of the crystal has little effect. The ultrasound modulated signal is not observed in this case. The bottom trace shows the signal observed in the presence of the reference 
beam, when two-wave mixing takes place. This lower trace is referred to as the envelope or "DC offset" signal. The amplitude of this signal is related to the magnitude of the ultrasound induced phase modulation. One can readily see that the PRC based interferometer, facilitated by the addition of a high voltage AC bias field, dramatically enhances the DC offset signal level.

In the current configuration, the diffracted reference beam is in phase with the transmitted signal beam such that the gain is real and thus we do not expect to see the 1 MHz modulation signal (see Eq. 3). In addition, a 500kHz low pass filter was employed in the detection system (see Fig. 2.), further reducing any $1-\mathrm{MHz}$ component in the detected signal. By using polarization optics we could also place the diffracted reference beam in quadrature with the transmitted signal beam. We then removed the low pass filter and still observed a $1 \mathrm{MHz}$ signal that was negligible with respect to the DC offset signal provided the reduced scattering coefficient of the sample was relatively high (typically greater than $1 \mathrm{~cm}^{-1}$ ). We believe that in diffusive media, the reduction of the 1 $\mathrm{MHz}$ modulated signal relative to the DC offset is due to the incoherent summation of signals associated with multiple optical paths possessing random phases. In other words, the $1 \mathrm{MHz}$ signal is not spatially coherent over the wavefront of the diffuse light and the signal modulation is greatly reduced when the scattered light is collected to a single large aperture detector.

The detected time-domain DC offset signal can be converted to a space-domain signal by multiplying the temporal coordinate by the speed of sound in the medium, as is typically done in B-mode ultrasound imaging. This yields a measure of the strength of acousto-optical interaction at points along the acoustic axis. This interaction is influenced 
by three factors: (1) the amplitude of the sound field, (2) the intensity of diffuse light, and (3) the optical characteristics of the medium. The acoustic pulse is a probe traveling down the acoustic axis, broadcasting information related to the acousto-optical interaction over that region of space for which the optical field possesses sufficient intensity to yield a detectable DC offset signal. For a focused ultrasound source, this region will likely be further restricted to the focal zone of the transducer. In the case of very short ultrasound pulses traversing optically uniform media, the duration of the DC offset signal simply defines the region of space where both the acoustical and optical fields have sufficient energy to produce a detectable signal. In the case of very long ultrasound pulses, the duration of the offset signal is essentially the duration of the acoustic pulse. ${ }^{12-13}$ The latter case is not particularly interesting from an imaging or tissue characterization perspective. The former allows for delineation of media variability along the ultrasound axis.

For media with spatial optical variability, these changes will be manifested as changes in the DC offset signal as the ultrasound probe beam traverses these inhomogeneities. Indeed, the ability to resolve spatial variations in optical properties along the ultrasound axis will be determined primarily by the spatial pulse length of the sound source. Sub-millimeter resolution in optical properties can thus be attained using standard diagnostic imaging transducers. Performance is enhanced by a combination of a very short acoustic pulse length and a uniformly illuminated diffusive medium. In our experiments, a beam expander is used to help ensure uniform illumination over the central region of the phantom. Therefore, the full-width at half maximum $(-6 \mathrm{~dB})$ duration of the detected DC offset signal $(\sim 11 \mu \mathrm{s})$, as shown in Fig. 5, is relatively large 
compared to the acoustic pulse length, which is about $2 \mu$ s. Any variability observed within this $11 \mu$ s signal will be the result of changes in media optical properties, most notably the absorption coefficient.

The ability to achieve spatial resolution along the ultrasound axis is illustrated in Fig. 6(a), which shows typical normalized DC offset signals obtained when the transducer is scanned at different locations with respect to the position of the optical absorber imbedded in the phantom. The upper (1), middle (2), and lower (3) plots correspond to the transducer axis positioned before, through, and after the optical absorber, respectively; each trace is displaced by $4.5 \mathrm{~mm}$ along the $\mathrm{X}$ axis. These locations are illustrated in Fig. 6(b) where the incident laser beam ( $\mathrm{Y}$ axis) is perpendicular to the page. When the ultrasound beam passes through the absorber, a peak is observed in the middle of the detected DC offset signal. The presence of this peak indicates that the acousto-optical interaction strength is temporarily diminished as the ultrasound passes through this point in space. The signals observed when the beam does not intersect the absorber (traces (1) and (3)) do not show this feature.

As previously mentioned, the spatial and temporal domains are related to each other through the speed of sound in the sample. Thereby, a time-domain DC offset signal corresponds to a spatial domain 1-D scan (an "A scan" in ultrasound imaging parlance) along the ultrasonic axis. When launching short ultrasound pulses through a uniform medium, the DC offset signal essentially tracks the local light distribution along the ultrasonic axis. If an optical absorber is present along ultrasound propagation path, it will absorb/trap any diffuse light passing through it locally, making it impossible for the modulated light to reach the detectors and yielding the image contrast observed 
experimentally. The spatial extent of the peak in Fig. 6(a-2) can be estimated by convolving the spatial envelope of the ultrasonic signal with the optical absorption profile along the ultrasound propagation direction. The full-width at half maximum (-6 dB) of the observed peak in Fig. 6(a-2) is on the order of $3.5 \mu$ s. As the speed of sound in the phantom is $1.5 \mathrm{~mm} / \mu \mathrm{s}$, our 1-D "image" of the optical inhomogeneity gives an absorber width of approximately $5.25 \mathrm{~mm}$ along the ultrasonic axis. By convolving the spatial envelope of the ultrasonic signal with the known spatial dimensions of the optical absorber, we find the full-width at half maximum of the convolution to be about $5.3 \mathrm{~mm}$, which agrees very well with the experimental result.

At position 2, shown in Fig. 6(b), different acoustic pulse lengths are used to image the absorber along the ultrasonic axis. The detected normalized DC offset signals using 1-cycle, 4-cycle and 6-cycle pulses to drive the ultrasound transducer are shown in Fig. 7. The signal contrast (i.e., relative change in the DC offset signal resulting from the absorber) decreases with increasing pulse duration. As the spatial pulse length gets long with respect to the absorber size, the pulse is no longer confined within the absorber at any point in space and the ability to distinguish the absorber from the background signal diminishes.

Enhanced resolution is possible through the use of higher frequency, broad band ultrasound transducers, which further localizes the ultrasound pulse in space. Our axial resolution does not change much from using a 1-cycle (Fig. 7(a)) to using a 2-cycle pulse to drive the transducer (Fig. 6(a-2)). This is due to the narrow-band nature of our transducer. The focal pressure responses from both the 1-cycle and the 2-cycle drive pulses have a comparable acoustic pulse widths of $2 \sim 3 \mu$ s as shown in Fig. 4(a) and Fig. 
4(b), which is the shortest pulse length our transducer can achieve. Typically, driving pulses in excess of 4-cycles have to be used in order for the focal pressure to reach its steady state as shown in Fig. 4(c). Ultrasound transducers used for biomedical imaging can produce pulses as short as 1.5 wavelengths, and could serve as ideal sound sources for probing the optical properties of diffuse media. Indeed, the fact that a PRC-based detection scheme allows for acousto-optical sensing with pulsed ultrasound suggests the possibility that conventional imaging machines could be utilized in dual-mode, ultrasound and acousto-optical imaging schemes. ${ }^{22}$

Using a 2-cycle pulse, an $\mathrm{X}$-axis scan of the transducer traversing the absorber was performed and a 2-D image was constructed corresponding to the $\mathrm{X}-\mathrm{Z}$ plane, where the Z-axis data derives from the time trace after converting to space using the known speed of sound in the medium. This 2-D image is shown in Fig. 8(a). In the middle section of Fig. 8(a), a black region can be readily seen, corresponding to the location of the optical absorber. The surrounding white area is the effective imaging area, where there is sufficient diffuse light to interact with the scanned ultrasound beam and generate a detectable signal. The striations in the image are due to the effect of environmental vibrations that are not completely compensated for by the PRC, and which lead to small variations in system sensitivity over the scan time. A close-up view of the image around the absorber is shown in Fig. 8(b). In this image, each scan line has been normalized to its maximum DC offset value to remove the effects of room vibration. Figure 8 demonstrates our ability to generate a 2-D image of an optical inhomogeneity by simply scanning the ultrasound transducer along one axis. The normalized acousto-optical amplitudes across the center of the optical absorber in both $X$ and $Z$ directions are shown in Fig. (9). The 
size of imaged absorber is comparable to that of the actual optical absorber $(5 \mathrm{~mm} \times 5 \mathrm{~mm})$ in both dimensions. If we define the imaging resolution by the distance required for a decrease in optical contrast from $90 \%$ to $10 \%$ of the maximum, the optical imaging resolutions along the $\mathrm{X}$ and $\mathrm{Z}$ directions are approximately $2 \mathrm{~mm}$ and $3 \mathrm{~mm}$, respectively, which are comparable to the lateral dimension of the focal region $(\sim 1.5 \mathrm{~mm})$ and the spatial ultrasonic pulse length $(\sim 3 \mathrm{~mm})$.

\section{CONCLUSIONS}

The photorefractive crystal based interferometry system has sufficient sensitivity to detect pulsed-ultrasound modulated light for imaging in optically diffuse tissue phantoms. The DC offset signal proves a mechanism for sensing and imaging in diffuse media and is dependent on the magnitude of the phase modulation and envelope of the pulse train, making detection of spatially incoherent phase modulation possible. The adaptive nature of the PRC makes the system somewhat insensitive to speckle decorrelation on the time scale of the crystal response time. However, under our experimental conditions, the response time of the crystal was $150 \mathrm{~ms}$ and some degree of vibration isolation was necessary. The use of a crystal with a faster response time could help to eliminate the speckle decorrelation problem altogether. By using short ultrasonic pulses, one-dimensional images of the targets positioned along the ultrasonic axis have been obtained through a single (averaged) time domain waveform; the axial resolution is determined by the spatial length of the ultrasound pulse. This fact makes high resolution attainable through the use of high frequency, broad band ultrasound transducers. Twodimensional imaging has also been demonstrated by mechanically scanning the transducer in one dimension. 


\section{ACKNOWLEDGEMENTS}

The authors would like to acknowledge Dr. Emmanuel Bossy, Dr. Florian Blonigen, Gopi Maguluri and Alex Nieva for valuable discussion of the results. This work was supported by CenSSIS, the Center for Subsurface Sensing and Imaging Systems, under the Engineering Research Centers Program of the National Science Foundation (award number EEC-9986821). 


\section{REFERENCES}

1. V. V. Tuchin, ed., Handbook of Optical Biomedical Diagnostics (SPIE, Washington, 2002).

2. L.-H. Wang, "Mechanisms of ultrasonic modulation of multiply scattered coherent light: an analytic model," Phys. Rev. Lett. 87, 043903-(1-4) (2001).

3. F. A. Marks, H. W. Tomlinson, and G. W. Brooksby, “A comprehensive approach to breast cancer detection using light: photon localization by ultrasound modulation and tissue characterization by spectral discrimination," in Photon Migration and Imaging in Random Media and Tissues, B. Chance and R. R. Alfano, eds., Proc. SPIE 1888, 500-510 (1993).

4. L.-H. Wang, S. L. Jacques, and X.-M. Zhao, "Continuous-wave ultrasonic modulation of scattered laser light to image objects in turbid media," Opt. Lett. 20, 629-631 (1995).

5. M. Kempe, M. Larionov, D. Zaslavsky, and A. Z. Genack, "Acousto-optic tomography with multiply scattered light,” J. Opt. Soc. Am. A 14, 1151-1158 (1997).

6. S. Leveque, A. C. Boccara, M. Lebec, and H. Saint-Jalmes, "Ultrasonic tagging of photon paths in scattering media: parallel speckle modulation processing," Opt. Lett. 24, 181-183 (1999).

7. M. Gross, P. Goy and M. Al-Koussa, "Shot-noise detection of ultrasound-tagged photons in ultrasound-modulated optical imaging," Opt. Lett. 28, 2482-2484 (2003). 
8. L.-H. Wang and G. Ku, "Frequency-swept ultrasound-modulated optical tomography of scattering media,” Opt. Lett. 23, 975-977 (1998).

9. G. Yao, S. Jiao and L.V. Wang, "Frequency-swept ultrasound-modulated optical tomography in biological tissue by use of parallel detection," Opt. Lett. 25, 734$736(2000)$.

10. B. C. Forget, F. Ramaz, M. Atlan, J. Selb, and A. C. Boccara, "High-contrast fast Fourier transform acousto-opticalal tomography of phantom tissues with a frequency-chirp modulation of the ultrasound," Appl. Opt. 42, 1379-1383 (2003).

11. A. Lev and B. G. Sfez, "Pulsed ultrasound-modulated light tomography," Opt. Lett. 28, 1549-1551 (2003).

12. L. Sui, T. Murray, G. Maguluri, A. Nieva, F. Blonigen, C. DiMarzio and R. A. Roy, "Enhanced detection of acousto-photonic scattering using a photorefractive crystal," in Photons Plus Ultrasound: Imaging and Sensing, A. A. Oraevsky and L. V. Wang, eds. Proc. SPIE 5320, 164-171 (2004).

13. T. W. Murray, L. Sui, G. Maguluri, R. A. Roy, A. Nieva, F. Blonigen, and C. A. DiMarzio, "Detection of ultrasound modulated photons in diffuse media using the photorefractive effect," Opt. Lett. 29, 2509-2511 (2004).

14. R. K. Ing and J.-P. Monchalin, "Broadband optical detection of ultrasound by two-wave mixing in a photorefractive crystal,” Appl. Phys. Lett. 59, 3233-3235 (1991).

15. A. Blouin and J.-P. Monchalin, "Detection of ultrasonic motion of a scattering surface by two-wave mixing in a photorefractive GaAs crystal,” Appl. Phys. Lett. 65, 932-934 (1994). 
16. T. W. Murray, H. Tuovinen, and S. Krishnaswamy, “Adaptive optical array receivers for detection of surface acoustic waves," Appl. Opt. 39, 3276-3284 (2000).

17. P. Delaye, A. Blouin, D. Drolet, L.-A. Montmorillon, G. Roosen, and J.-P. Monchalin, "Detection of ultrasonic motion of a scattering surface by photorefractive InP:Fe under an applied dc field,” J. Opt. Soc. Am. B 14, 1723 1734 (1997).

18. J. E. Millerd, M. Ziari and A. Partovi, "Photorefractivity in semiconductors," in Nonlinear Optics in Semiconductors I (Vol. 58 of Semiconductors and Semimetals), E. Garmire and A. Kost, eds. (Academic, San Diego, Calif., 1998), pp. 319-401.

19. P. Delaye, L.-A. Montmorillon, and G. Roosen, "Transmission of time modulated optical signals through an absorbing photorefractive crystal,” Opt. Comm. 118, 154-164 (1995).

20. L. Solymar, D. J. Webb and A. Grunnet-Jepsen, The Physics and Applications of Photorefractive Materials (Oxford, New York, 1996).

21. F. A. Duck, Physical Properties of Tissue (Academic, San Diego, 1990).

22. E. Bossy, L. Sui, T. W. Murray, and R. A. Roy, "Combination of B-mode imaging and acousto-photonic sensing using a commercial ultrasound scanner," J. Acoust. Soc. Am. 115, 2523 (2004). 


\section{FIGURE CAPTIONS}

Fig. 1. The two wave mixing (TWM) configuration of a PRC with length L: The signal beam before the PRC is given by $\mathrm{I}_{\mathrm{SO}}$ the intensity after the two wave mixing process is given by $\mathrm{I}_{\mathrm{SE}}$.

Fig. 2. Experimental setup for PRC based detection of ultrasound modulated optical signals: FG- function generator, A- power amplifier, M- impedance matching box, TStranslation stage, UT- ultrasound transducer, VBS- variable beamsplitter, RB- reference beam, SB- signal beam, BE- beam expander, BP- optical bandpass filter, APD- avalanche photodiode, PA- preamplifier, LP- lowpass filter.

Fig. 3. A photograph of a phantom $(4 \mathrm{~cm} \times 4 \mathrm{~cm})$ with an optical absorber $(5 \mathrm{~mm} \times 5 \mathrm{~mm})$ imbedded in the middle. The reduced scattering coefficient of the phantom is approximately $2 \mathrm{~cm}^{-1}$ and the absorption coefficient of the absorber is about $3 \mathrm{~cm}^{-1}$.

Fig. 4. Normalized focal pressure responses generated when driving the ultrasound transducer with (a) 1-cycle, (b) 2-cycle, and (c) 4-cycle electrical pulses at a $1 \mathrm{MHz}$ center frequency.

Fig. 5. Experimental results showing the detected optical signals sensed without the PRC based system (top) and with the PRC based system (bottom). 
Fig. 6. The normalized DC offset signals (a) detected when the transducer is scanned along the $\mathrm{X}$ axis at 3 different locations corresponding to: position 1, 2 and 3 in (b). There is a $4.5-\mathrm{mm}$ spacing between these 3 locations with position 2 passing through the middle of the absorber.

Fig. 7. The effect of changing the ultrasound pulse length on the DC offset component of the ultrasound modulated optical signal.

Fig. 8. Two-dimensional image (X-Z plane) obtained by scanning the transducer across a $5 \mathrm{~mm} \times 5 \mathrm{~mm}$ optical absorber: (a) global view; (b) zoomed-in view centered on the absorber.

Fig. 9. Normalized 1-D acousto-optical image contrast obtained along orthogonal lines intersecting at the center of the optical absorber. Acousto-optical contrast for paths in the $\mathrm{X}$ (“scanning") and Z (“axial") directions are given in plots (a) and (b), respectively. 
Fig. 1

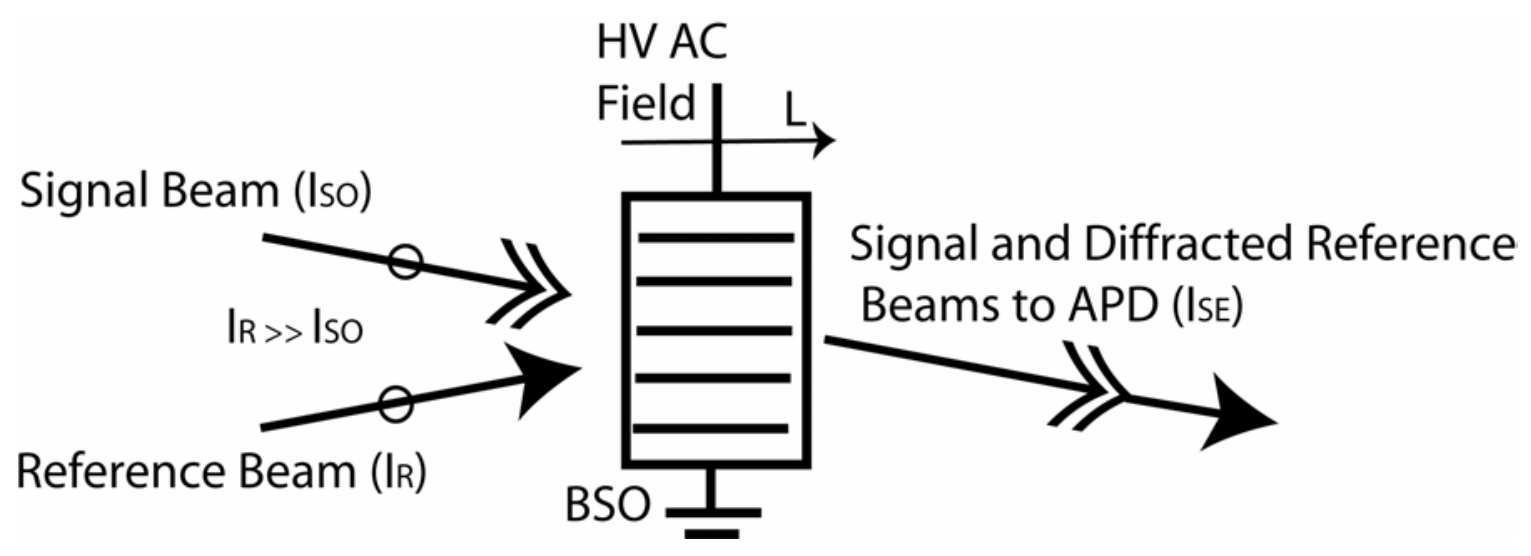


Fig. 2

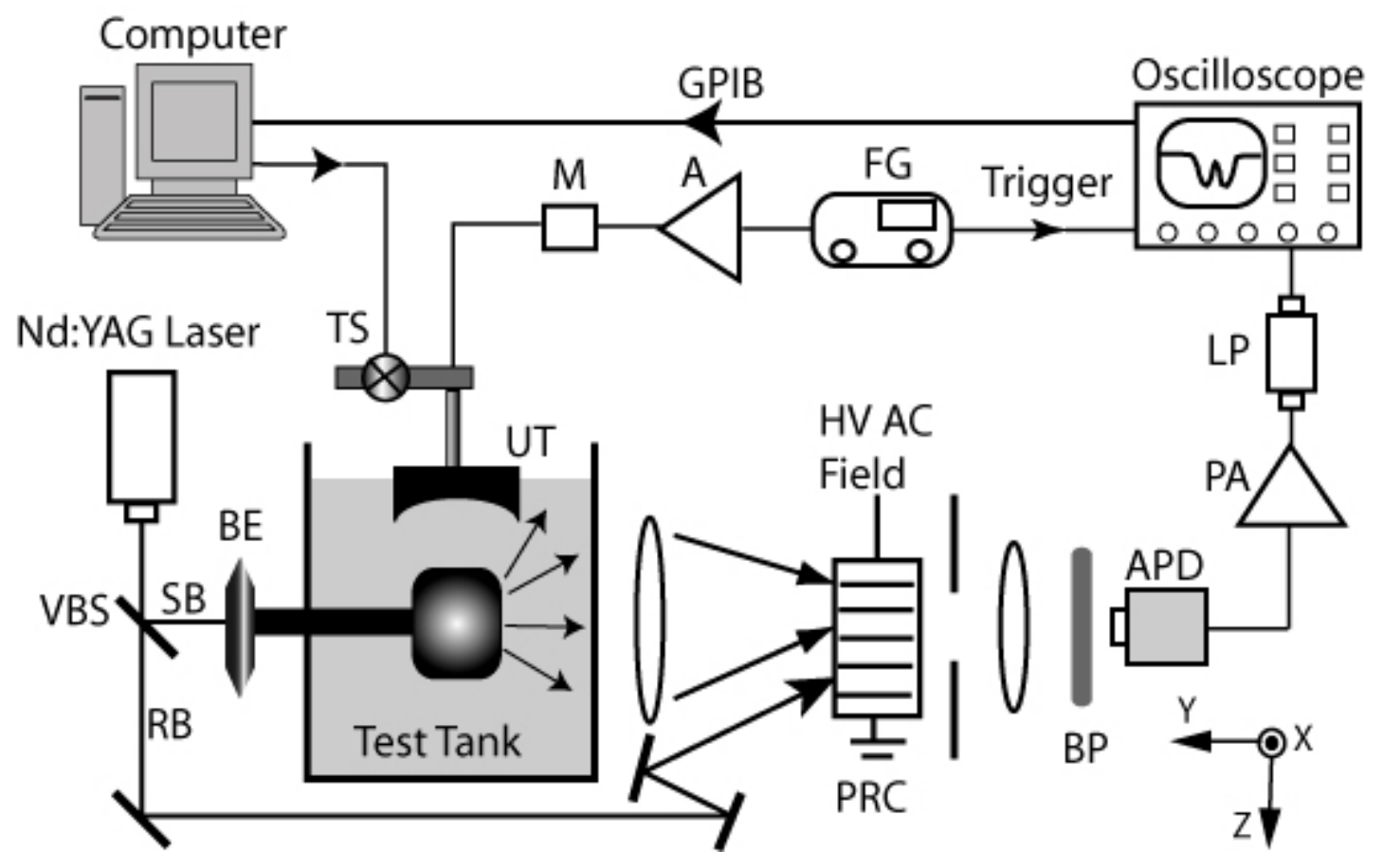


Fig. 3

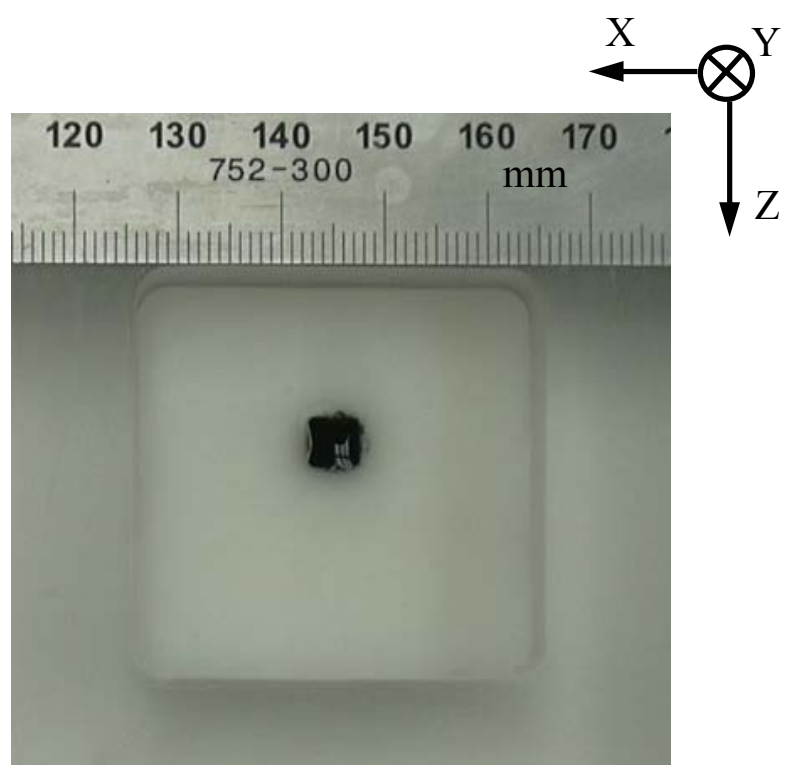


Fig. 4

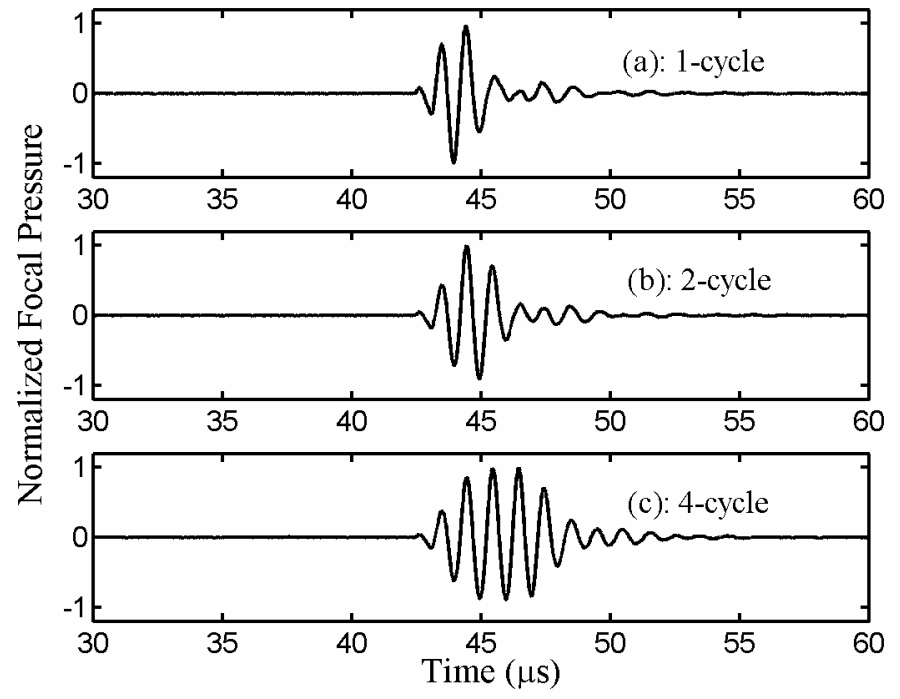


Fig. 5

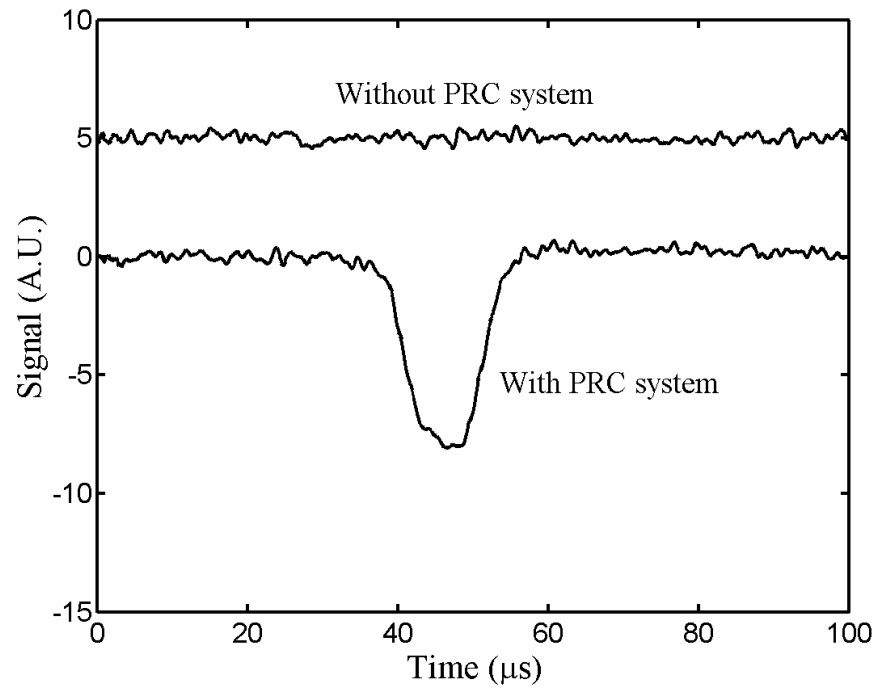


Fig. 6

(a)

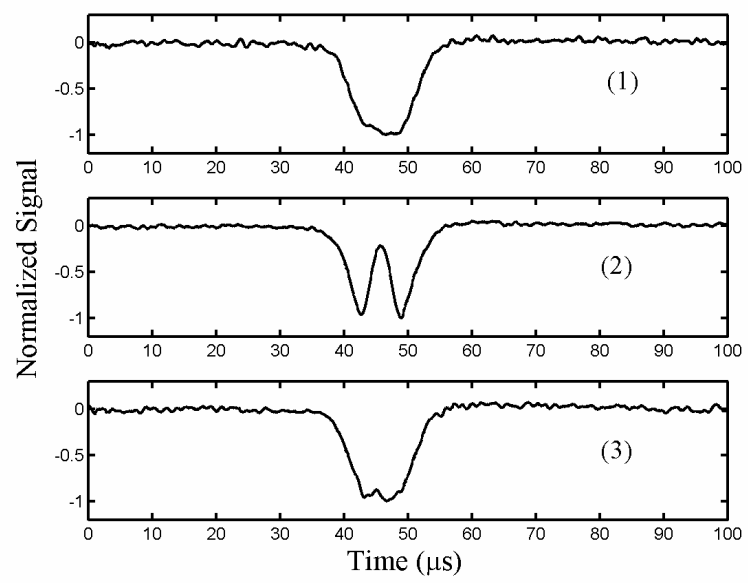

(b)

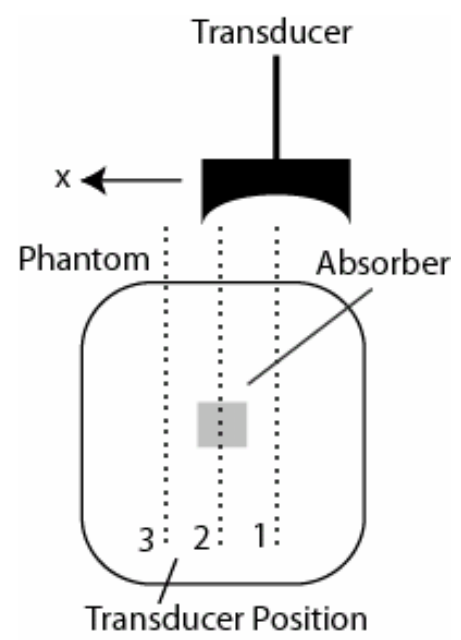


Fig. 7

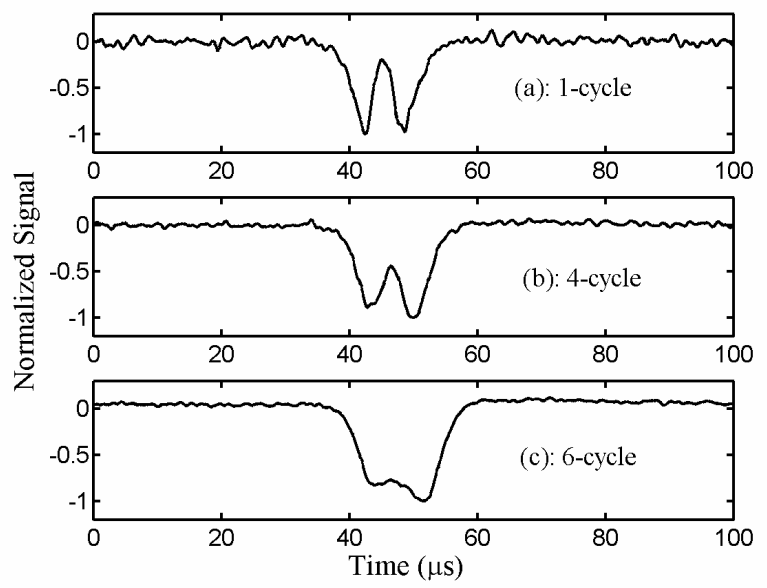


Fig. 8

(a)

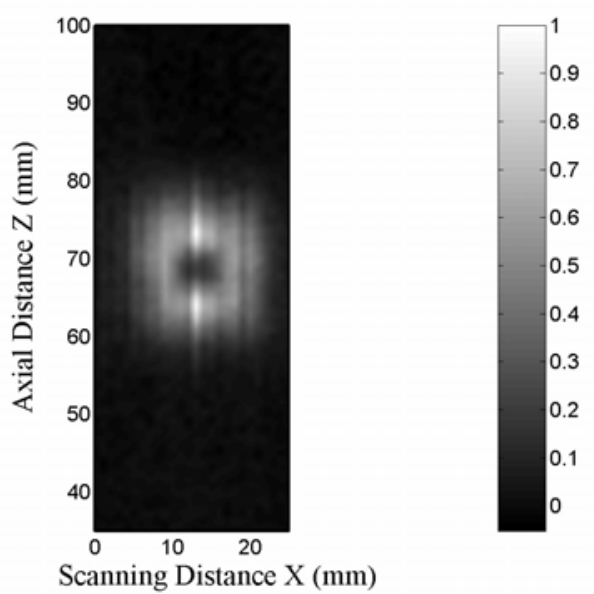

(b)

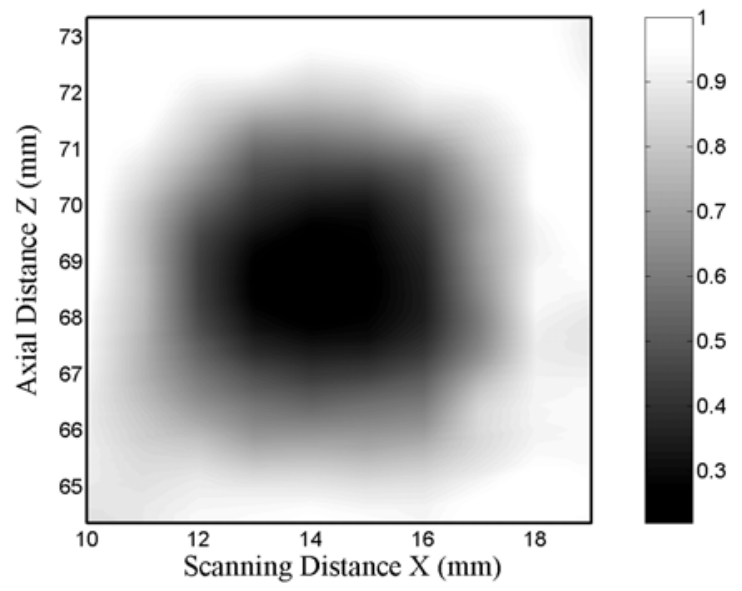


Fig. 9

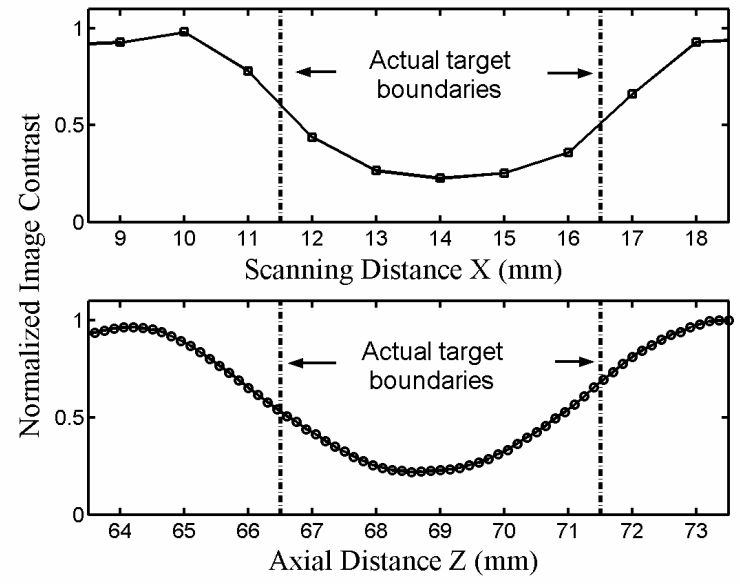

